OPEN ACCESS

Edited by:

Iris Chi,

University of Southern California,

United States

Reviewed by:

Ferdinando Toscano,

University of Bologna, Italy

Igor Linkov,

United States Army Crops of

Engineers, United States

*Correspondence: Maria Velana velana@ifado.de

Specialty section: This article was submitted to Psychology for Clinical Settings, a section of the journal

Frontiers in Psychology

Received: 12 May 2021 Accepted: 24 June 2021

Published: 19 July 2021

Citation:

Velana M and Rinkenauer G (2021) Individual-Level Interventions for Decreasing Job-Related Stress and Enhancing Coping Strategies Among

Nurses: A Systematic Review.

Front. Psychol. 12:708696 doi: 10.3389/fpsyg.2021.708696

\section{Individual-Level Interventions for Decreasing Job-Related Stress and Enhancing Coping Strategies Among Nurses: A Systematic Review}

\author{
Maria Velana* and Gerhard Rinkenauer \\ Department of Ergonomics, Leibniz Research Center for Working Environment and Human Factors (IfADo) at Dortmund \\ University of Technology, Dortmund, Germany
}

Background: Nurses are facing unprecedented amounts of pressure because of the ongoing global health challenges. Improving nurses' resilience to job-related stress and enhancing their strategies to cope effectively with stressors are key issues facing many health care institutions during the COVID-19 pandemic. This literature review aimed to: a) provide a thorough overview of individual-level interventions for stress management among nurses, b) identify measurement tools utilized to evaluate nurses' stress level, and c) provide the best evidence-based recommendations for future research and practice adapted to the current restrictions.

Design: Systematic review.

Data Sources: Studies published between January 2000 and October 2020 were retrieved from the following sources: EBSCOhost, Dortmund University Library, PubMed, Medline, Google Scholar, Applied Nursing Research, and reference lists from relevant articles.

Review methods: Individual-level interventions with a control group or a placebo intervention were included in the final sample. Primary outcome was defined as a change in individual stress level or stress symptoms which were measured by objective or subjective instruments with evidence of validity. Articles published in English or German were included in the present review.

Results: In total, 27 relevant studies were included into the current review. There are some indications that technology-delivered interventions with relaxation and stress management interventions comprising cognitive-behavioral components might be effective in decreasing stress among nurses and improving their well-being. Furthermore, although there were some attempts to collect objectively measured parameters for assessing the primary outcome of stress, the majority of the interventions utilized self-reported stress scales.

Conclusion: A wide range of interventions are available for nurses. However, it is of utmost importance to develop and implement stress management programs that are conveniently accessible in the workplace and above all, meet the current restrictions for minimizing human contacts. To this end, innovative interventions delivered through 
digital technology, such as virtual reality, seem to be a promising solution for combating the detrimental impact of stress on nurses. Special attention should be also paid to applying standardized objective measurement tools to allow the assessment of sensitive physiological indices and the generalizability of scientific knowledge.

Keywords: nurses, job-related stress, stress management, coping strategies, technology-delivered interventions, objective measurement tools

\section{INTRODUCTION}

Nowadays, nurses are facing unprecedented amounts of pressure not only due to the growing global health demands but also the current COVID-19 pandemic. The rapid spread of the virus during the last year has placed a huge burden on many health care systems scrambling to cover the needs for intensive care unit beds, personal protective equipment for both health care professionals and patients, and offer high-quality health services to their end-users (Arnetz et al., 2020). The current pandemic outbreak can be considered as a stressor that has significantly affected nurses' mental health. According to stress literature, when the existence of an organism is threatened by exposure to either a physiological or a psychological stressor, the system reacts to the stressful situation in a generalized, complex fashion (Matousek et al., 2010). The transactional model of stress postulates that one's perception of a stressor will depend on the degree to which an individual assesses this stressor as meaningful and relevant to them in a given context (Lazarus and Folkman, 1984). Further, meaningfulness will shape the strength of one's reaction to the event. On physiological level, stress triggers an initial activation of the sympathetic nervous and adrenomedullary systems resulting in increases in cardiac activity (Zhang et al., 2018). Furthermore, activation of the hypothalamus-pituitary-adrenal (HPA) axis provokes endocrine and immune changes leading to the release of cortisol and cytokines in an effort to re-establish body balance (Matousek et al., 2010). Stress research has showed that stressful events at the workplace can cause high physiological and psychological workload which can lead, in turn, to serious health problems and burnout [Lazarus and Folkman, 1984; Eatough et al., 2011; Velana and Rinkenauer (in press)].

In stressful occupations, such as nursing, the experience of stress deriving from lack of social support, heavy workload, conflicts with colleagues and other critical job-related factors is strongly linked with poor health (Tyler and Cushway, 1995). In fact, scientific evidence supports such an association, as stress in nurses can cause health problems and psychosomatic disorders, absenteeism, workplace injuries and errors related to patient care (Shirey, 2006). Moreover, study findings have indicated that nursing profession is associated with high rates of psychiatric outpatient referrals and suicide (Jones, 1987). More recently, in a cross-sectional study among 1257 Chinese health care providers from 34 national hospitals, female nurses, especially those who work in Wuhan, reported more severe symptoms on distress, anxiety and depression as compared to physicians (Lai et al., 2020). Another cross-sectional study that was conducted in
Germany, indicated that nurses reported higher levels of stress and subjective burden as well as lower levels of job satisfaction and experienced support than physicians (Kramer et al., 2020). In a broader context, the present health crisis urges attention on nurses' mental health and on the strategies that should be developed to enhance their well-being and quality of life. Therefore, developing and implementing innovative approaches may be a best practice policy to reduce stress and improve health (Hatcher et al., 2006).

Improving resilience to stress and enhancing nurse's strategies to respond effectively to stressors are key issues facing many health care institutions during the COVID-19 pandemic. Resilience is considered one's ability to recover easily and quickly from adverse circumstances that happen over the course of their life (Zautra et al., 2010). Applying this notion to an organizational environment, resilience to stress implies that, in general, employees can respond in a productive way when encounter significant job-related changes or pressure to reach outcomes (Home and Orr, 1998). In nursing profession, this can be proved particularly challenging, as nurses often have to deal with human suffering, interpersonal difficulties and other job-related issues such as bullying and violence (Jackson et al., 2007), conditions that are associated with high levels of stress and demand adequate personal resources and coping strategies. Hence, enhancing nurses' resources and support may have the potential to develop their capacities to deal with stress and workplace adversities. The last years health care organizations around the globe have developed and implemented various individual-level interventions and strategies to empower employees to tackle setbacks at the workplace. In particular, interest has been growing in highlighting the effects of interventions on stress management and improving nurses' mental health, such as mindfulness, meditation and relaxation techniques (Delgado et al., 2017; Ghawadra et al., 2019). Furthermore, in his study, Cottrell (2001) showed that focused interventions for mental health nurses can enhance work-related factors, such as job satisfaction, and ameliorate stressors at workplace. Another literature review revealed that different stress management programs, such as training in therapeutic skills or in behavioral techniques, may help nurses address stress (Edwards and Burnard, 2003). Nevertheless, the rigor of a number of studies has induced methodological weaknesses related, for example, to measurement tools utilized, study sample size or statistical methods used for the analysis of their results. Although many interventions appear promising to effectively decrease stress and improve well-being among nurses, there is another body of evidence that indicates only moderate or no 
intervention effects, and calls for further research in this field (Chesak et al., 2019; Li et al., 2019). In light of the current contact restrictions, it still remains unclear what strategies would be suitable to tackle job-related stress throughout the COVID-19 crisis and the era after it.

Since nurses experience high levels of stress at the workplace, it is of vital importance to review and systematically evaluate the studies that utilized various individual-level strategies as a method to reduce their stress. Therefore, the present literature review aims to address the following issues: (a) to provide a thorough overview of the stress management interventions targeted at helping nurses develop skills to cope effectively with stress, (b) to identify measurement tools utilized to evaluate nurses' stress level (i.e., subjective and objectively measured parameters), and (c) to provide future research and practice with fruitful evidence-based directions adapted to the current restrictions.

\section{METHODS}

\section{Search Strategies}

In an effort to examine the current state of the science regarding individual-level interventions for reducing job-related stress in nurses, studies published between January 2000 and October 2020 were retrieved from the following sources: EBSCOhost, Dortmund University Library, PubMed, Medline, Google Scholar, Applied Nursing Research, and reference lists from relevant articles. Although job-related stress in nurses and their coping strategies has drawn researchers' attention for over 30 years (e.g., Jones, 1987; Tyler and Cushway, 1995), the authors decided to focus on the advances of the last two decades so that they would provide the readers with a thorough insight about the topic and at the same time, suggest up-to-date directions for future studies that would align with the present global challenges. Specific search strategies were developed for each database to identify relevant interventions for this literature review. The present literature search was performed utilizing the following key terms: nurses, stress reduction interventions, stress management programs, (workplace) mental health interventions, job stress and coping strategies, in various combinations. Particularly, the following keyword combinations were applied: nurses AND stress reduction interventions, nurses AND stress management programs AND job stress, nurses AND mental health interventions AND job stress AND coping strategies, nurses AND workplace mental health interventions AND job stress. Special attention was paid to the differences among the databases in regard to vocabulary and syntax rules. The search was performed in November 2020.

The review protocol included two main steps: the first step involved reviewing of databases, while the second step consisted of identifying and screening all relevant papers according to inclusion and exclusion criteria. In order to ensure consistency and rigor, the guideline of "The Preferred Reporting Items for Systematic Reviews and Meta-Analyses (PRISMA)" was utilized (Moher et al., 2009).

\section{Inclusion Criteria}

According to research literature, nurses are more likely to experience higher levels of job-related stress compared to other hospital employees and health professionals (Moustaka and Constantinidis, 2010; Golshiri et al., 2012; Kramer et al., 2020). Although stressful events and emergency situations seem to be common phenomena for hospital employees, nurses are prone to stress because of the psychological, physical and social attributes embedded in their occupational sector (Moustaka and Constantinidis, 2010). Building on this notion, only empirical research articles that focused on nurses who work in health care facilities, aiming at stress reduction were included in the final sample. Furthermore, two individual-level interventions should have been compared to each other, or an individual-level intervention should have been be compared to a control or placebo group in a prospective way. For instance, randomized controlled studies with or without random assignment, studies with quasi-experimental design and preposttest design studies with control group and/or placebo cohort were considered for further evaluation. Studies were also considered for further analysis, if the components of the stress management intervention, such as methods, frequency and duration of the intervention, were clearly described. Primary outcome was defined as a change in individual stress level or stress symptoms which were measured by objective or subjective instruments with evidence of validity. Secondary outcomes could be, but not limited to: burnout, depression, anxiety, quality of life, job satisfaction, etc. Articles published in English or German were included in the present review.

\section{Exclusion Criteria}

Interventions that focused not only on nurses (e.g., nursing students, nurse aids) or studies that included other health care professionals were excluded from this review. Other exclusion criteria were pure qualitative studies, one group pre-post designs, studies aiming at organization level changes and articles published earlier than 2000.

\section{Data Extraction}

Data were extracted and formatted based on the review aim utilizing a pre-defined data extraction worksheet in Excel. Table 1 summarizes all relevant characteristics of identified studies. Particularly, the headings include: (1) study characteristics (author, year of publication, place of study, sample size and setting), and (2) intervention characteristics (design, duration and components, measurement tools, follow-up, and main findings). This process was checked by the two review authors.

\section{RESULTS}

\section{Search Outcome}

Based on inclusion and exclusion criteria, first the two review authors independently screened the titles and abstracts of all relevant articles. Next, full-text versions of all potentially eligible articles were evaluated independently by the two authors to define whether all inclusion criteria were met. External experts would be consulted to achieve a consensus in case 
TABLE 1 | Summary of articles included in the current literature review.

\begin{tabular}{|c|c|c|c|c|c|c|}
\hline $\begin{array}{l}\text { Author (year) } \\
\text { and place }\end{array}$ & $\begin{array}{l}\text { Sample size and } \\
\text { setting }\end{array}$ & Design & $\begin{array}{l}\text { Duration and } \\
\text { components }\end{array}$ & Measurement tools & Follow-up & Main findings \\
\hline $\begin{array}{l}\text { Alkhawaldeh et al. } \\
\text { (2020), Jordan }\end{array}$ & $\begin{array}{l}\text { Total }(N=184): \\
\text { Treatment }(n=92) ; \\
\text { Control }(n=92) ; \text { CHCC }\end{array}$ & Cluster-RCT & $\begin{array}{l}\text { 2-week SMIP (six } \\
\text { 2-hour sessions } \\
\text { twice a day) } \\
\text { Waitlist control } \\
\text { group }\end{array}$ & NSS Brief COPE Scale & $\begin{array}{l}\text { Baseline, after the } \\
\text { intervention and } \\
\text { follow-up } 2 \\
\text { months after the } \\
\text { intervention }\end{array}$ & $\begin{array}{l}\downarrow \text { stress after the } \\
\text { intervention and at follow-up } \\
(p=0.001) \uparrow \text { coping } \\
\text { strategies after intervention } \\
\text { and at follow-up }(p=0.001)\end{array}$ \\
\hline $\begin{array}{l}\text { Bernburg et al. } \\
\text { (2019), Germany }\end{array}$ & $\begin{array}{l}\text { Total }(N=86): \\
\text { Treatment }(n=44) ; \\
\text { Control }(n=42) ; \\
\text { Psychiatric hospitals }\end{array}$ & $\begin{array}{l}\text { Randomized } \\
\text { controlled pilot } \\
\text { study }\end{array}$ & $\begin{array}{l}\text { 12-week mental } \\
\text { health program } \\
\text { (1.5-2 } \mathrm{h} / \text { week }) \\
\text { Waitlist control } \\
\text { group }\end{array}$ & $\begin{array}{l}\text { PSQ ERSQ-27 BRCS } \\
\text { SWOP-K9 QRI } \\
\text { Evaluation form }\end{array}$ & $\begin{array}{l}\text { Baseline and three } \\
\text { follow-ups over a } \\
\text { period of } 36 \\
\text { weeks (after } 3 \\
\text { months, T1; after } 6 \\
\text { months, T2; after } \\
12 \text { months, T3) }\end{array}$ & $\begin{array}{l}\downarrow \text { stress at T1 }(p<0.01), \mathrm{T} 2 \\
(p<0.01) \text { and T3 }(p<0.01) \\
\uparrow \text { for all additional outcomes } \\
\text { at T1, T2and T3 }(p<0.05) \\
\uparrow \text { program evaluation }\end{array}$ \\
\hline $\begin{array}{l}\text { Bernburg et al. } \\
\text { (2020), Germany }\end{array}$ & $\begin{array}{l}\text { Total }(N=94) \text {; } \\
\text { Treatment }(n=47) ; \\
\text { Control }(n=47) ; \\
\text { Hospital }\end{array}$ & $\mathrm{RCT}$ & $\begin{array}{l}\text { 12-week } \\
\text { work-related } \\
\text { self-care skill } \\
\text { training ( } 1.5 \\
\text { h/week) Waitlist } \\
\text { control group }\end{array}$ & $\begin{array}{l}\text { PSQ COPSOQ MBI-EE } \\
\text { ERSQ-27 Evaluation } \\
\text { form }\end{array}$ & $\begin{array}{l}\text { Baseline and three } \\
\text { follow-ups over a } \\
\text { period of } 36 \\
\text { weeks (after } 3 \\
\text { months, T1; after } 6 \\
\text { months, T2; after } \\
12 \text { months, T3) }\end{array}$ & $\begin{array}{l}\downarrow \text { stress at T1 }(p<0.001), \\
\text { T2 } p<0.001) \text { and T3 }(p< \\
0.01) \uparrow \text { emotional } \\
\text { exhaustion and emotion } \\
\text { regulation skills at T1, } \\
\text { T2and T3 }(p<0.05) \uparrow \text { job } \\
\text { satisfaction at T1 }(p=0.01) \\
\uparrow \text { program evaluation }\end{array}$ \\
\hline $\begin{array}{l}\text { Calder Calisi } \\
\text { (2017), USA }\end{array}$ & $\begin{array}{l}\text { Total }(N=46): \\
\text { Treatment }(n=24) ; \\
\text { Control }(n=22) ; \\
\text { General Hospital }\end{array}$ & $\begin{array}{l}\text { Randomized, } \\
\text { waitlist control } \\
\text { design }\end{array}$ & $\begin{array}{l}\text { 8-week RR } \\
\text { (45-min session; } \\
\text { self-practice } \\
\text { 10-20 min twice a } \\
\text { day) Waitlist } \\
\text { control group }\end{array}$ & $\begin{array}{l}\text { STAI Semantic } \\
\text { differential scales }\end{array}$ & $\begin{array}{l}\text { Baseline and after } \\
\text { the intervention }\end{array}$ & $\begin{array}{l}\downarrow \text { anxiety }(p=0.02) \text { and } \\
\text { stress }(p=0.003) \uparrow \\
\text { confidence in teaching RR } \\
(p<0.001)\end{array}$ \\
\hline $\begin{array}{l}\text { Collier et al. } \\
\text { (2018), USA and } \\
\text { UK }\end{array}$ & $\begin{array}{l}\text { Total }(N=16) \text { : } \\
\text { Treatment }(n=8) ; \\
\text { Control }(n=8) ; \\
\text { Psychiatric inpatient } \\
\text { unit (Hospital and } \\
\text { Mental Health Services) }\end{array}$ & Randomized trial & $\begin{array}{l}\text { 4-week MSET } \\
\text { (two 40-min } \\
\text { sessions/week) } \\
\text { Control group; } \\
\text { standard unit } \\
\text { lounge }\end{array}$ & $\begin{array}{l}\text { Pulse rate STAI POMS } \\
\text { Evaluation form }\end{array}$ & $\begin{array}{l}\text { Before and after } \\
\text { each session }\end{array}$ & $\begin{array}{l}\downarrow \text { pulse rate }(p=0.001) \text {, in } \\
\text { State scale }(p<0.001) \text { and } \\
\text { Trait scale }(p=0.015) \uparrow \\
\text { Confusion Bewilderment } \\
\text { sub-scale of POMS }(p= \\
0.004) \uparrow \text { program evaluation }\end{array}$ \\
\hline $\begin{array}{l}\text { Ghawadra et al. } \\
\text { (2020), Malaysia }\end{array}$ & $\begin{array}{l}\text { Total }(N=249: \\
\text { Treatment }(n=123) ; \\
\text { Control }(n=126) ; \\
\text { Teaching hospital }\end{array}$ & $\mathrm{RCT}$ & $\begin{array}{l}\text { 4-week } \\
\text { mindfulness- } \\
\text { based intervention } \\
\text { (2-hour workshop; } \\
\text { self-practice } \\
\text { guided by a } \\
\text { website) Waitlist } \\
\text { control group }\end{array}$ & DASS-21 JSS MAAS & $\begin{array}{l}\text { Baseline, after the } \\
\text { intervention and } \\
\text { follow-up } 8 \text { weeks } \\
\text { after the } \\
\text { intervention }\end{array}$ & $\begin{array}{l}\downarrow \text { stress }(p<0.001), \text { anxiety } \\
(p=0.001) \text { and depression } \\
(p<0.001) \text { over time } \uparrow \\
\text { mindfulness }(p<0.001) \\
\text { over time } \uparrow \text { job satisfaction } \\
(p<0.001)\end{array}$ \\
\hline $\begin{array}{l}\text { Gollwitzer et al. } \\
\text { (2018), Germany }\end{array}$ & $\begin{array}{l}\text { Total }(N=129) \text { : } \\
\text { Treatment (MCII, } n= \\
41 \text {; IIMCII, } n=41) ; \\
\text { Control }(n=47) ; \text { Health } \\
\text { institutions }\end{array}$ & $\begin{array}{l}\text { Randomized } \\
\text { factorial design }\end{array}$ & $\begin{array}{l}\text { 3-week MCII } \\
\text { (mental exercise } \\
\text { daily) 3-week } \\
\text { IIMCII (modified } \\
\text { mental exercise } \\
\text { daily) Passive } \\
\text { control group }\end{array}$ & $\begin{array}{l}\text { PSQ-20 Physical } \\
\text { symptoms subscale of } \\
\text { BOSS II UWES-9 }\end{array}$ & $\begin{array}{l}\text { Baseline and after } \\
\text { the intervention }\end{array}$ & $\begin{array}{l}\downarrow \text { stress in the MCll group } \\
\text { compared to the control } \\
\text { group ( } p=0.019) \uparrow \text { work } \\
\text { engagement in the MCll } \\
\text { group as compared to the } \\
\text { IIMCI ( } p=0.029 \text { ) and the } \\
\text { control group }(p=0.046)\end{array}$ \\
\hline
\end{tabular}


TABLE 1 | Continued

\begin{tabular}{|c|c|c|c|c|c|c|}
\hline $\begin{array}{l}\text { Author (year) } \\
\text { and place }\end{array}$ & $\begin{array}{l}\text { Sample size and } \\
\text { setting }\end{array}$ & Design & $\begin{array}{l}\text { Duration and } \\
\text { components }\end{array}$ & Measurement tools & Follow-up & Main findings \\
\hline $\begin{array}{l}\text { Hersch et al. } \\
\text { (2016), USA }\end{array}$ & $\begin{array}{l}\text { Total }(N=104) \text { : } \\
\text { Treatment }(n=52) ; \\
\text { Control }(n=52) ; \\
\text { Hospital }\end{array}$ & $\mathrm{RCT}$ & $\begin{array}{l}\text { 12-week } \\
\text { web-based } \\
\text { BREATHE } \\
\text { program (unlimited } \\
\text { online access) } \\
\text { Waitlist control } \\
\text { group }\end{array}$ & $\begin{array}{l}\text { NSS Symptoms of } \\
\text { Distress Coping with } \\
\text { Stress WLQ Use of } \\
\text { Substances for Stress } \\
\text { Relief Drinking Quantity } \\
\text { and Frequency } \\
\text { Understanding } \\
\text { Depression and Anxiety } \\
\text { Nurses Job } \\
\text { Satisfaction Scale }\end{array}$ & $\begin{array}{l}\text { Baseline and after } \\
\text { the intervention }\end{array}$ & $\begin{array}{l}\downarrow \text { stress }(p<0.001) \text { and } \\
\text { NSS sub-scales }(p<0.05) \\
\text { apart from sub-scale lack of } \\
\text { support No effect on } \\
\text { additional outcomes }\end{array}$ \\
\hline $\begin{array}{l}\text { Hsieh et al. (2020), } \\
\text { Taiwan }\end{array}$ & $\begin{array}{l}\text { Total }(N=135) \text { : } \\
\text { Treatment (BT, } n=49 ; \\
\text { SDBT, } n=47) \text { Control } \\
\text { ( } n=39) ; \text { Psychiatric } \\
\text { wards }\end{array}$ & $\begin{array}{l}\text { Quasi- } \\
\text { experimental } \\
\text { study }\end{array}$ & $\begin{array}{l}\text { 6-week BT (1 } \\
\text { h/week) 6-week } \\
\text { SDBT (once a } \\
\text { week) Waitlist } \\
\text { control group }\end{array}$ & $\begin{array}{l}\text { CES-D OSI-2 RS } \\
\text { Physiological } \\
\text { parameters (HRV: } \\
\text { SDNN, LF, HF; RR) } \\
\text { Rehabilitation strength } \\
\text { chart Simplified health } \\
\text { scale }\end{array}$ & $\begin{array}{l}\text { Baseline and after } \\
\text { the intervention }\end{array}$ & $\begin{array}{l}\downarrow \text { stress }(p=0.013) \text { in SDBT } \\
\text { group } \uparrow \text { depressive } \\
\text { symptoms }(p<0.001) \text {, } \\
\text { resilience }(p<0.001) \text {, and } \\
\text { respiration rate for BT ( } p< \\
0.001 \text { ) and SDBT ( } p= \\
0.002)\end{array}$ \\
\hline $\begin{array}{l}\text { Kurebayashi et al. } \\
\text { (2012), Brazil }\end{array}$ & $\begin{array}{l}\text { Total }(N=75) \text { : } \\
\text { Treatment (Needle } \\
\text { group, } n=27 \text {; Seed } \\
\text { group, } n=26) \text {; Control } \\
(n=22) ; \text { Teaching } \\
\text { hospital }\end{array}$ & $\mathrm{RCT}$ & $\begin{array}{l}\text { 8-week } \\
\text { auriculotherapy } \\
\text { with needles (eight } \\
\text { sessions, 5-10 } \\
\text { min/week) 8-week } \\
\text { auriculotherapy } \\
\text { with seeds (eight } \\
\text { sessions, 5-10 } \\
\text { min/week) Passive } \\
\text { control group }\end{array}$ & $\begin{array}{l}\text { LSS Folkman and } \\
\text { Lazarus' Ways of } \\
\text { Coping questionnaire }\end{array}$ & $\begin{array}{l}\text { Baseline, after } 4 \\
\text { sessions, after } 8 \\
\text { sessions and } \\
\text { follow-up } 15 \text { days } \\
\text { after the } \\
\text { intervention }\end{array}$ & $\begin{array}{l}\downarrow \text { stress after } 8 \text { sessions ( } p \\
=0.020 \text { ) and at follow-up ( } p \\
=0.003 \text { ) in the needle } \\
\text { group } \uparrow \text { Distancing domain } \\
(p=0.039 \text { ) and Confrontive } \\
\text { Coping domain }(p=0.029) \\
\text { at follow-up in the needle } \\
\text { group } \uparrow \text { Seeking Social } \\
\text { Support domain }(p=0.022) \\
\text { after } 8 \text { sessions in the seed } \\
\text { group }\end{array}$ \\
\hline $\begin{array}{l}\text { Lary et al. (2019), } \\
\text { Iran }\end{array}$ & $\begin{array}{l}\text { Total }(N=70) \text { : } \\
\text { Treatment }(n=35) ; \\
\text { Control }(n=35) ; \\
\text { Teaching hospital }\end{array}$ & $\begin{array}{l}\text { Quasi- } \\
\text { experimental } \\
\text { study }\end{array}$ & $\begin{array}{l}\text { 6-week } \\
\text { McNamara } \\
\text { educational } \\
\text { method (1 h/week) } \\
\text { Waitlist Control } \\
\text { group }\end{array}$ & SRI & $\begin{array}{l}\text { Baseline, after the } \\
\text { intervention and } \\
\text { follow-up } 8 \text { weeks } \\
\text { after the } \\
\text { intervention }\end{array}$ & $\begin{array}{l}\downarrow \text { stress }(p=0.021) \text { over } \\
\text { time }\end{array}$ \\
\hline $\begin{array}{l}\text { Lin et al. (2019), } \\
\text { China }\end{array}$ & $\begin{array}{l}\text { Total }(N=90): \\
\text { Treatment }(n=44) ; \\
\text { Control }(n=46) ; \\
\text { General hospital }\end{array}$ & $\begin{array}{l}\text { Randomized } \\
\text { controlled design }\end{array}$ & $\begin{array}{l}\text { Modified 8-week } \\
\text { MBSR program } \\
\text { (group sessions } 2 \\
\text { h/week and } \\
\text { home-based } \\
\text { practice } 20 \text { min } \times \\
6 \text { days/week) } \\
\text { Waitlist control } \\
\text { group }\end{array}$ & $\begin{array}{l}\text { PSS PANAS CD-RISC } \\
\text { MMSS }\end{array}$ & $\begin{array}{l}\text { Baseline, after the } \\
\text { intervention (T1), } \\
\text { and follow-up } 3 \\
\text { months later (T2) }\end{array}$ & $\begin{array}{l}\downarrow \text { stress and negative affect } \\
\text { at T1 }(p<0.01) \text { and T2 }(p< \\
0.05) \text { respectively } \uparrow \text { positive } \\
\text { affect at T1 and T2 }(p< \\
0.05) \text { and resilience at T2 ( } p \\
<0.05) \text { No effect on job } \\
\text { satisfaction }\end{array}$ \\
\hline $\begin{array}{l}\text { McElligott et al. } \\
\text { (2003), USA }\end{array}$ & $\begin{array}{l}\text { Total }(N=20): \\
\text { Treatment }(n=12) ; \\
\text { Control }(n=8) ; \\
\text { University Hospital }\end{array}$ & $\begin{array}{l}\text { Quasi- } \\
\text { experimental } \\
\text { design }\end{array}$ & $\begin{array}{l}\text { 4-week AMMA } \\
\text { therapy ( } 1 \text { h/week) } \\
\text { Control group; } \\
\text { 4-week STTP }\end{array}$ & $\begin{array}{l}\text { Physiologic Parameters } \\
\text { (blood pressure, heart } \\
\text { rate, pulse oximetry, } \\
\text { and skin temperature) } \\
\text { VAS Evaluation } \\
\text { interview } \\
\text { questionnaires }\end{array}$ & $\begin{array}{l}\text { Baseline, before } \\
\text { and after each } \\
\text { treatment, and at } \\
\text { completion of the } \\
\text { study }\end{array}$ & $\begin{array}{l}\downarrow \text { anxiety over time No effect } \\
\text { on physiologic parameters } \uparrow \\
\text { program evaluation }\end{array}$ \\
\hline
\end{tabular}


TABLE 1 | Continued

\begin{tabular}{|c|c|c|c|c|c|c|}
\hline $\begin{array}{l}\text { Author (year) } \\
\text { and place }\end{array}$ & $\begin{array}{l}\text { Sample size and } \\
\text { setting }\end{array}$ & Design & $\begin{array}{l}\text { Duration and } \\
\text { components }\end{array}$ & Measurement tools & Follow-up & Main findings \\
\hline $\begin{array}{l}\text { Moeini et al. } \\
\text { (2011), Iran }\end{array}$ & $\begin{array}{l}\text { Total }(N=58) \text { : } \\
\text { Treatment }(n=29) ; \\
\text { Control }(n=29) ; \\
\text { Training hospital }\end{array}$ & $\begin{array}{l}\text { Quasi- } \\
\text { experimental } \\
\text { study }\end{array}$ & $\begin{array}{l}\text { 3-week cognitive- } \\
\text { behavioral } \\
\text { program based on } \\
\text { PRECEDE model } \\
\text { (five } 60-90 \text { min } \\
\text { sessions) Passive } \\
\text { control group }\end{array}$ & $\begin{array}{l}\text { NSS Questionnaire } \\
\text { based on PRECEDE } \\
\text { model Evaluation form }\end{array}$ & $\begin{array}{l}\text { Baseline and } \\
\text { follow-up } 1.5 \\
\text { months after the } \\
\text { intervention }\end{array}$ & $\begin{array}{l}\downarrow \text { stress }(p<0.001) \uparrow \\
\text { PRECEDE model constructs } \\
\text { and stress management } \\
\text { behaviors }(p<0.001)\end{array}$ \\
\hline $\begin{array}{l}\text { Nazari et al. } \\
(2015) \text {, Iran }\end{array}$ & $\begin{array}{l}\text { Total }(N=66) \text { : } \\
\text { Treatment }(n=33) ; \\
\text { Control }(n=33) ; \text { ICUs } \\
\text { (Hospital) }\end{array}$ & $\mathrm{RCT}$ & $\begin{array}{l}\text { 4-week massage } \\
\text { therapy ( } 25 \text {-min } \\
\text { sessions twice a } \\
\text { week) Passive } \\
\text { control group }\end{array}$ & OSI & $\begin{array}{l}\text { Baseline, after the } \\
\text { intervention and } \\
\text { follow-up } 2 \text { weeks } \\
\text { after the } \\
\text { intervention }\end{array}$ & $\begin{array}{l}\downarrow \text { stress }(p<0.001) \text { and } \\
\text { subscale scores }(p<0.05) \\
\text { over time }\end{array}$ \\
\hline $\begin{array}{l}\text { Niva et al. (2020), } \\
\text { India }\end{array}$ & $\begin{array}{l}\text { Total }(N=30): \\
\text { Treatment }(n=15) ; \\
\text { Control }(n=15) ; \\
\text { Tertiary care hospital }\end{array}$ & $\mathrm{RCT}$ & $\begin{array}{l}\text { Mahamantra } \\
\text { chanting } \\
\text { intervention for } 45 \\
\text { days (20 min/day) } \\
\text { Passive control } \\
\text { group }\end{array}$ & $\begin{array}{l}\text { Stress biomarkers } \\
\text { (Serum cortisol, } \\
\text { DHEA-S, SAA) } \\
\text { Biochemical } \\
\text { parameters (Glucose } \\
\text { and lipid profile) }\end{array}$ & $\begin{array}{l}\text { Baseline and } \\
\text { follow-up after } 2 \\
\text { menstrual cycles } \\
\text { after the } \\
\text { intervention }\end{array}$ & $\begin{array}{l}\downarrow \text { serum cortisol }(p= \\
0.012), \text { SAA level }(p=0.04), \\
\text { glucose }(p=0.001), \text { HbA1c } \\
(p=0.041) \text {, total cholesterol } \\
(p<0.001), \text { LDLc }(p< \\
0.001) \text { and TGL }(p=0.17) \uparrow \\
\text { HDLc }(p=0.033)\end{array}$ \\
\hline $\begin{array}{l}\text { Orly et al. (2012), } \\
\text { Israel }\end{array}$ & $\begin{array}{l}\text { Total }(N=36) \text { : } \\
\text { Treatment }(n=20) ; \\
\text { Control }(n=16) ; \\
\text { Hospital }\end{array}$ & $\begin{array}{l}\text { Pre-posttest } \\
\text { design study with } \\
\text { control }\end{array}$ & $\begin{array}{l}\text { 16-week CBI } \\
\text { course (4 h/week) } \\
\text { and five } \\
\text { job-related 3-hour } \\
\text { seminars Control } \\
\text { group; five } \\
\text { job-related 3-hour } \\
\text { seminars }\end{array}$ & SOC PSS POMS & $\begin{array}{l}\text { Baseline and after } \\
\text { the intervention }\end{array}$ & $\begin{array}{l}\downarrow \text { stress }(p<0.05) \text { and } \\
\text { POMS fatigue }(p<0.05) \uparrow \\
\text { SOC }(p<0.05) \text { and POMS } \\
\text { vigor }(p<0.05)\end{array}$ \\
\hline $\begin{array}{l}\text { Palumbo et al. } \\
\text { (2012), USA }\end{array}$ & $\begin{array}{l}\text { Total }(N=14) \text { : } \\
\text { Treatment }(n=7) ; \\
\text { Control }(n=7) ; \\
\text { Hospital }\end{array}$ & $\mathrm{RCT}$ & $\begin{array}{l}\text { 15-week Tai Chi } \\
\text { program (group } \\
\text { practice } 45 \\
\text { min/week and } \\
\text { self-practice at } \\
\text { least } 10 \text { min } \times 4 \\
\text { days /week) } \\
\text { Passive control } \\
\text { group }\end{array}$ & $\begin{array}{l}\text { SF-36 Health Survey } \\
\text { NSS PSS } \\
\text { Sit-and-reach test } \\
\text { Isometric knee } \\
\text { extensor strength test } \\
\text { dynamometer } \\
\text { Functional reach test } \\
\text { Nordic Musculoskeletal } \\
\text { Questionnaire WLQ } \\
\text { Work absenteeism }\end{array}$ & $\begin{array}{l}\text { Baseline and after } \\
\text { the intervention }\end{array}$ & $\begin{array}{l}\text { No effect on stress } \uparrow \text { work } \\
\text { productivity }(p=0.03) \text { and } \\
\text { functional reach }(p<0.01)\end{array}$ \\
\hline $\begin{array}{l}\text { Singh and Jain } \\
\text { (2017), India }\end{array}$ & $\begin{array}{l}\text { Total }(N=40): \\
\text { Treatment }(n=20) ; \\
\text { Control }(n=20) ; \\
\text { Hospital }\end{array}$ & $\begin{array}{l}\text { Pre-posttest } \\
\text { design with control }\end{array}$ & $\begin{array}{l}\text { Self-help } \\
\text { intervention (four } \\
30 \text {-min sessions } \\
\text { with an interval of } \\
10 \text { days) Passive } \\
\text { control group }\end{array}$ & $\begin{array}{l}\text { Psychosocial Stress } \\
\text { Questionnaire } \\
\text { Occupational Stress } \\
\text { Index }\end{array}$ & $\begin{array}{l}\text { Baseline and after } \\
\text { the intervention }\end{array}$ & $\begin{array}{l}\downarrow \text { occupational stress and in } \\
\text { psychosocial stress }(p< \\
\text { 0.01) }\end{array}$ \\
\hline $\begin{array}{l}\text { Villani et al. (2013), } \\
\text { Italy }\end{array}$ & $\begin{array}{l}\text { Total }(N=30) \text { : } \\
\text { Treatment }(n=15) ; \\
\text { Control }(n=15) ; \\
\text { Oncology hospital }\end{array}$ & $\begin{array}{l}\text { Between-subjects } \\
\text { design }\end{array}$ & $\begin{array}{l}\text { 4-week M-SIT } \\
\text { (15-min sessions } \\
\text { twice a week) } \\
\text { Control group; } \\
\text { neutral stimuli } \\
\text { (15-min sessions } \\
\text { twice a week) }\end{array}$ & $\begin{array}{l}\text { MSP STAI COPE-4 } \\
\text { JCQ }\end{array}$ & $\begin{array}{l}\text { Baseline, before } \\
\text { and after each } \\
\text { session, after the } \\
\text { intervention }\end{array}$ & $\begin{array}{l}\uparrow \text { state anxiety }(p<0.001) \text {, } \\
\text { trait anxiety }(p=0.041) \text { and } \\
\text { coping skills acquisition }(p< \\
0.05)\end{array}$ \\
\hline
\end{tabular}


TABLE 1 | Continued

\begin{tabular}{|c|c|c|c|c|c|c|}
\hline $\begin{array}{l}\text { Author (year) } \\
\text { and place }\end{array}$ & $\begin{array}{l}\text { Sample size and } \\
\text { setting }\end{array}$ & Design & $\begin{array}{l}\text { Duration and } \\
\text { components }\end{array}$ & Measurement tools & Follow-up & Main findings \\
\hline $\begin{array}{l}\text { Walker (2006), } \\
\text { USA }\end{array}$ & $\begin{array}{l}\text { Total }(N=98) \text { : } \\
\text { Treatment }(n=58) ; \\
\text { Control }(n=40) ; \\
\text { Hospital }\end{array}$ & $\begin{array}{l}\text { Quasi- } \\
\text { experimental } \\
\text { design }\end{array}$ & $\begin{array}{l}\text { 4-week HRTT } \\
\text { HeartTouch } \\
\text { technique (3-hour } \\
\text { educational } \\
\text { session; } \\
\text { self-practice; } \\
\text { 1-hour session } 2 \\
\text { weeks after the } \\
\text { initial session; final } \\
\text { follow-up session) } \\
\text { Control group } \\
\text { (2-hour } \\
\text { educational } \\
\text { session; final } \\
\text { follow-up session) }\end{array}$ & $\begin{array}{l}\text { PSS SWB DRS Diary } \\
\text { HeartTouch } \\
\text { questionnaire }\end{array}$ & $\begin{array}{l}\text { Baseline and after } \\
\text { the intervention }\end{array}$ & $\begin{array}{l}\downarrow \text { stress }(p<0.001) \text {, and } \uparrow \\
\text { hardiness }(p<0.001) \text { and } \\
\text { spiritual well-being }(p< \\
0.05) \text { in the treatment group } \\
\downarrow \text { stress }(p<0.001) \text { and } \uparrow \\
\text { hardiness }(p<0.05) \text { in the } \\
\text { control group }\end{array}$ \\
\hline $\begin{array}{l}\text { Yang et al. (2018), } \\
\text { China }\end{array}$ & $\begin{array}{l}\text { Total }(N=100) \text { : } \\
\text { Treatment }(n=50) ; \\
\text { Control }(n=50) ; \\
\text { Psychiatric } \\
\text { departments }\end{array}$ & $\begin{array}{l}\text { Pre-posttest } \\
\text { design with control }\end{array}$ & $\begin{array}{l}\text { 8-week MBSR } \\
\text { therapy (once a } \\
\text { week; either group } \\
\text { training or } \\
\text { home-based } \\
\text { practice) Control } \\
\text { group; routine } \\
\text { psychological } \\
\text { support }\end{array}$ & SCL-90 SDS SAS NSS & $\begin{array}{l}\text { Baseline and after } \\
\text { the intervention }\end{array}$ & $\begin{array}{l}\downarrow \text { stress, anxiety and } \\
\text { depression scores }(p< \\
0.001) \uparrow \text { mental health }(p< \\
0.001)\end{array}$ \\
\hline
\end{tabular}

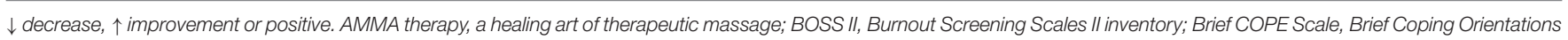

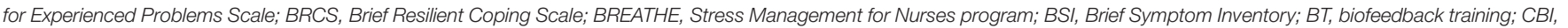

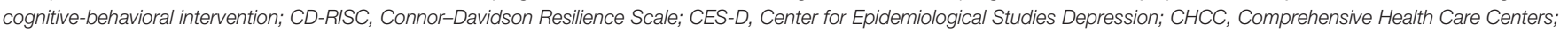

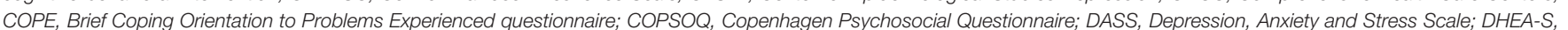

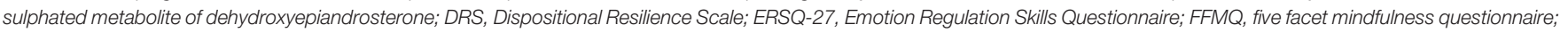

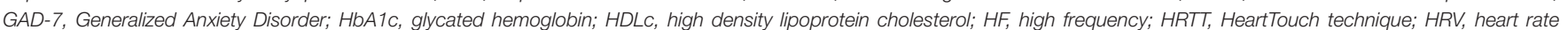

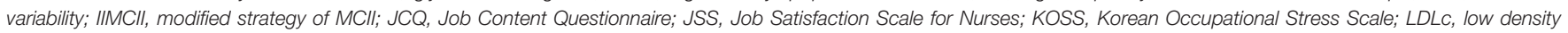

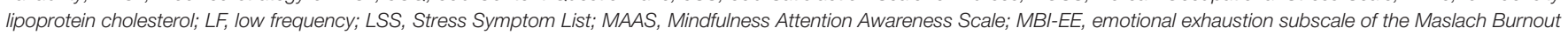

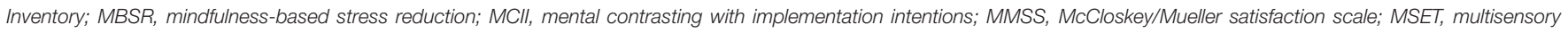

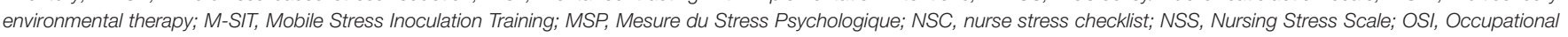

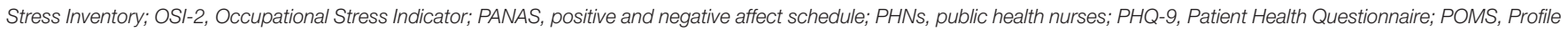

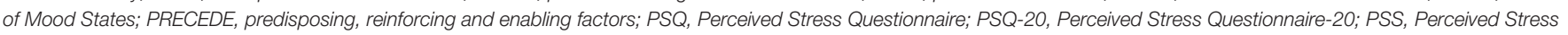

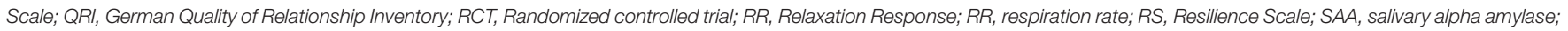

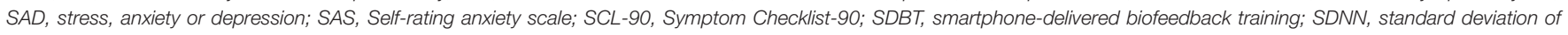

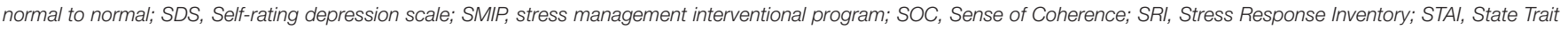

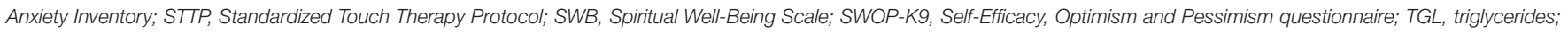
UWES-9, Utrecht Work Engagement Scale; VAS, Visual Analog Scale; WHOQOL-BREF, World Health Organization Quality-of-Life Scale; WLQ, Work Limitations Questionnaire.

of disagreement. However, this was not the case for the current review.

In total, records 5,931 were retrieved. Additionally, three studies were identified from the reference lists of previously published literature reviews (Chesak et al., 2019; Ghawadra et al., 2019; Bakker et al., 2020). 5,892 records were left for screening after removing duplicates. Next, titles and abstracts were assessed and 5,833 were excluded, leaving 59 potentially relevant articles. Screening of the full-text articles indicated that 32 did not fulfill the inclusion criteria, leaving 27 studies for this literature review. Figure 1 tracks the selection process of the relevant studies utilizing a modified version of the PRISMA flow diagram (Moher et al., 2009).

\section{STUDY CHARACTERISTICS}

\section{Place of Study}

Four of these studies were carried out in European countries, and the other studies were from different countries: Brazil $(n=2)$, China $(n=2)$, India $(n=2)$, Iran $(n=4)$, Israel $(n=1)$, Jordan $(n$ $=1)$, Korea $(n=1)$, Malaysia $(n=1)$, Taiwan $(n=2)$, USA $(n=$ 


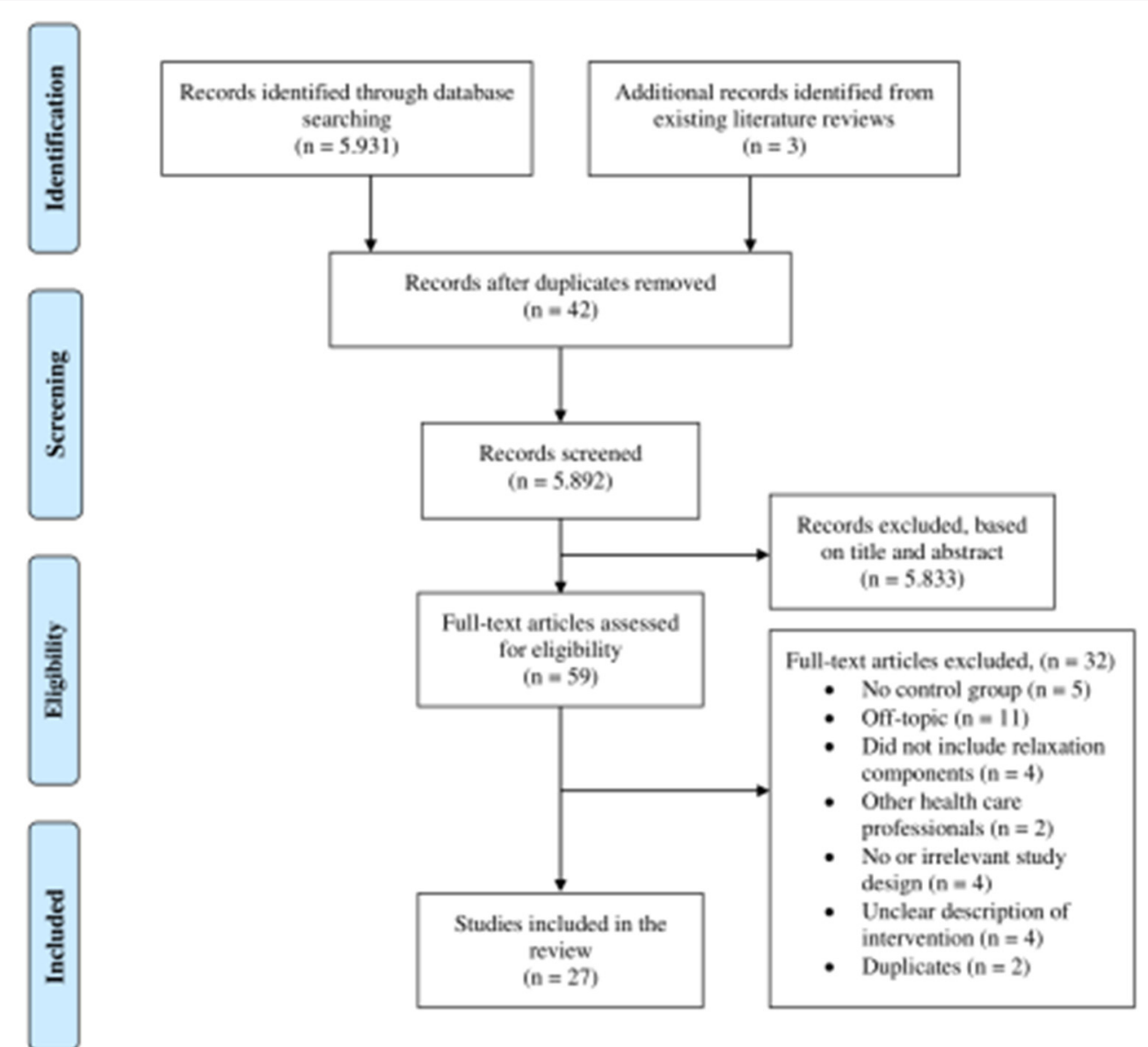

FIGURE 1 | Search strategy for the inclusion and exclusion of articles based on a modified version of PRISMA flow diagram (Moher et al., 2009).

6). Furthermore, one study took place in health care institutions based in two different countries, namely the USA and the UK.

\section{Sample Size and Setting}

The sample size of the included studies varied widely, from 14 participants (Palumbo et al., 2012) to 249 participants (Ghawadra et al., 2020). Participant work settings included hospitals (McElligott et al., 2003; Cohen-Katz et al., 2005; Walker, 2006; Moeini et al., 2011; Kurebayashi et al., 2012; Orly et al., 2012; Palumbo et al., 2012; Villani et al., 2013; Hersch et al., 2016; Bahmanzadeh and Haji Alizadeh, 2017; Calder Calisi, 2017; Singh and Jain, 2017; Wang et al., 2017; Prado et al., 2018; Hwang and Jo, 2019; Lary et al., 2019; Lin et al., 2019; Bernburg et al., 2020; Ghawadra et al., 2020; Niva et al., 2020), settings that focus on mental health (Collier et al., 2018; Yang et al., 2018; Bernburg et al., 2019; Hsieh et al., 2020), intensive care units (Nazari et al., 2015), and health care institutions with different organizational and hierarchical structures (Gollwitzer et al., 2018; Alkhawaldeh et al., 2020).

\section{INTERVENTION CHARACTERISTICS}

\section{Design}

Fifteen studies used a randomized controlled trial to study the effects of the treatment on nurses' stress level (Kurebayashi et al., 2012; Palumbo et al., 2012; Nazari et al., 2015; Hersch et al., 2016; Calder Calisi, 2017; Collier et al., 2018; Gollwitzer et al., 2018; Prado et al., 2018; Bernburg et al., 2019, 2020; Hwang and Jo, 2019; Lin et al., 2019; Alkhawaldeh et al., 2020; Ghawadra et al., 2020; Niva et al., 2020). However, only three studies employed blind procedures (Prado et al., 2018; Alkhawaldeh et al., 2020; Bernburg et al., 2020). The rest of these studies either did not use blinding procedures or did not report any attempts of blinding. Seven studies used a quasi-experimental design (McElligott et al., 2003; Walker, 2006; Moeini et al., 2011; Bahmanzadeh and Haji Alizadeh, 2017; Wang et al., 2017; Lary et al., 2019; Hsieh et al., 2020) and the remaining five studies utilized a pre-posttest design with control group (Cohen-Katz et al., 2005; Orly et al., 2012; Villani et al., 2013; Singh and Jain, 2017; Yang et al., 2018). Out of the 27 studies included in the review, only eight studies conducted a psychological screening 
to define whether participants suffered from moderate or high levels of psychological stress prior to being invited to take part in the research study (Kurebayashi et al., 2012; Villani et al., 2013; Nazari et al., 2015; Singh and Jain, 2017; Prado et al., 2018; Yang et al., 2018; Ghawadra et al., 2020; Niva et al., 2020).

\section{Intervention Duration and Components}

All the interventions included in the current review aimed at treatment of the individual. The intervention duration ranged from 2 weeks (Alkhawaldeh et al., 2020) to 16 weeks (Orly et al., 2012). As for the modalities, the most common interventions were technology-delivered interventions for stress management and mental health. Particularly, three studies implemented self-help programs guided by a website (Hersch et al., 2016; Gollwitzer et al., 2018; Ghawadra et al., 2020) and three additional interventions investigated the effectiveness of mobile phonedelivered programs for stress management (Villani et al., 2013; Hwang and Jo, 2019; Hsieh et al., 2020). Other commonly used modality types included mindfulness-based programs (CohenKatz et al., 2005; Walker, 2006; Wang et al., 2017; Yang et al., 2018; Lin et al., 2019), cognitive-behavioral interventions (Moeini et al., 2011; Orly et al., 2012; Bahmanzadeh and Haji Alizadeh, 2017), self-care interventions (Singh and Jain, 2017; Lary et al., 2019; Alkhawaldeh et al., 2020), auriculotherapy (Kurebayashi et al., 2012; Prado et al., 2018), massage (McElligott et al., 2003; Nazari et al., 2015), psychological competence trainings combined with cognitive-behavioral components (Bernburg et al., 2019, 2020), breathing exercises (Calder Calisi, 2017), chanting mantras (Niva et al., 2020), physical activity training (Palumbo et al., 2012) and multisensory environmental therapy (Collier et al., 2018).

\section{Measurement Tools and Follow-Up}

Diverse instruments were used depending on the research aims of each study. The present review focuses on the subjective and objective assessment tools for measuring stress in nurses. Among the 27 articles, 18 different instruments were utilized to assess subjective stress experience. The most commonly used tools to record stress were the Perceived Stress Scale (PSS) and the Nursing Stress Scale (NSS), which were used in 10 studies. Other instruments that were frequently utilized, were the Perceived Stress Questionnaire (PSQ), the Stress Symptom List (LSS), the State Trait Anxiety Inventory (STAI), and the Depression, Anxiety, and Stress Scale (DASS). Furthermore, the primary outcome of stress was also objectively measured in four interventions. In particular, physiological parameters, such as blood pressure, pulse oximetry, skin temperature, respiration rate and cardiac response, were assessed to quantify stress level (McElligott et al., 2003; Collier et al., 2018; Hsieh et al., 2020). One study evaluated serum stress markers and metabolic parameters without utilizing self-report (Niva et al., 2020).

In these studies, assessments of the primary outcome were performed for all participants before the beginning of the intervention, at baseline. All the interventions repeated the stress assessment after the completion of the treatment. However, only in 11 interventions, the measurements were conducted for a longer period of time, following the participants after the end of the treatment (Cohen-Katz et al., 2005; Kurebayashi et al., 2012;
Nazari et al., 2015; Wang et al., 2017; Prado et al., 2018; Bernburg et al., 2019, 2020; Lary et al., 2019; Lin et al., 2019; Alkhawaldeh et al., 2020; Ghawadra et al., 2020). The end point for these follow-up examinations ranged from 2 weeks (Kurebayashi et al., 2012; Nazari et al., 2015; Prado et al., 2018) to 36 weeks (Bernburg et al., 2019, 2020) after the intervention.

\section{Main Findings}

Twenty-three studies showed that stress level decreased after the intervention. However, two studies measured perceived stress only either with Visual Analog Scale [VAS; (McElligott et al., 2003)] or State Trait Anxiety Inventory [STAI; (Collier et al., 2018)]. Although one intervention mentioned decrease in selfreported measures, they did not report $p$ values (McElligott et al., 2003). Furthermore, four studies did not indicate changes in perceived stress (Cohen-Katz et al., 2005; Palumbo et al., 2012; Villani et al., 2013; Wang et al., 2017). On physiological level, it was shown that the treatment had a significant effect on objectively measured stress indices (Collier et al., 2018; Hsieh et al., 2020; Niva et al., 2020). Nevertheless, one study reported no changes in the measured physiological parameters (McElligott et al., 2003). Overall, nine studies indicated long-term decrease in perceived work-related stress (Kurebayashi et al., 2012; Nazari et al., 2015; Prado et al., 2018; Bernburg et al., 2019, 2020; Lary et al., 2019; Lin et al., 2019; Alkhawaldeh et al., 2020; Ghawadra et al., 2020).

\section{DISCUSSION}

This systematic review of the literature investigating individual-level interventions for stress management in nurses revealed a wide variety of programs that can be mainly classified into: (a) technology-based interventions for stress management and mental health either guided by a website or delivered through mobile phones, (b) mindfulness-based and spiritual interventions, (c) programs with cognitivebehavioral components, and (d) programs addressing body. In particular, there are some indications that technologydelivered interventions with relaxation components and stress management interventions comprising self-care skills, cognitivebehavioral components and relaxation might be effective in reducing stress among nurses and improving their mental well-being. In this direction, earlier research reviews have indicated that a wide range of interventions are available and seem promising for decreasing psychological distress (Delgado et al., 2017; Ghawadra et al., 2019; Bakker et al., 2020). Although evidence supports the effectiveness of these mechanisms in tackling stress, the rapid changes in health care systems and the unpreceded pressure that nurses experience, highlight the need to develop interventions adapted to the new overwhelming demands. Indeed, the prevalence of mental health problems among health care workers, especially during and after outbreaks, is high (Ricci-Cabello et al., 2020). Furthermore, these problems are usually associated with long-term mental health burden and thus, hinder the immediate response to health threats, such as the present COVID-19 crisis. 
It is of utmost importance to develop and implement stress management interventions that are not only conveniently accessible in the workplace but also, they meet the strict conditions for minimizing human contacts. To this end, evidence-based interventions and self-care practices for those in immediate need delivered through digital technology seem to be a promising solution for combating the detrimental psychological and physiological impact among nurses. For example, there is recently an emerging body of studies that examines the implementation of a self-help virtual reality (VR) protocol to overcome the negative consequences of the quarantine by reliving stress among individuals (Riva and Riva, 2020; Riva et al., 2020). The protocol is designed to simulate a natural environment, while the user can perform daily exercises aiming at self-concertation and relaxation. By transferring this idea to a demanding workplace, where restrictions for social contact apply, it might be an effective way to enhance nurses' resilience and generally, improve their mental health. Therefore, future research will be needed to examine, if the continuous use of technologybased stress management and the refinement of its technological capabilities would lead to individually tailored self-help programs and to a more positive effect.

Furthermore, the most commonly utilized instrument for assessing the primary outcome of stress was self-reported scales. However, the current review of the literature identified some efforts from these interventions to include objectively measured parameters in order to explore the effect of strategies developed on physiological level. The experience of high job stress can cause alternations in the physiological processes that the human body mobilizes in an attempt to re-establish inner balance. In psychophysiological studies, the exposure to a stressful event is associated with intense cardiac activity (Johnston et al., 2016) which may be considered as a predisposing factor for the onset of lifestyle diseases, such as depression and heart disease. Additionally, the prolonged activation of the HPA axis can lead to increased concentrations of stress hormones harming the immune system (Aguilera, 2011; Herman et al., 2016).

The assessment of specific stress biomarkers could provide considerable advantages to future studies: such measures will allow researchers to define the outcomes of their interventions in a more systematic and precise way, taking into consideration the individual differences. It is well-known that people respond to potential stressors with great variability (McEwen, 1998). In this regard, diverse individual-related factors, such as gender, age, health status and personality characteristics, may regulate not only physiological reactivity to stress but also individuals' ability to activate their resources and cope with challenges. By capturing physiological responses, it would be possible to reveal which psychophysiological mechanisms are involved into resilience processes and how individual characteristics are interwoven in physiological traits. This may contribute, in turn, to a better understanding of human body and the implementation of effective stress management strategies based on objective indices. Moreover, concrete physiological outcomes can be directly associated with stress scales that may have implications for individual's health status, and can reduce confounding effects by response bias inherent in self-report ratings (Schnall et al.,
1992; Bosma et al., 1997; McEwen, 1998). Therefore, future studies could utilize physiological parameters as indices for assessing their effectiveness to reduce stress and overcoming certain methodological issues of self-report.

There are some other issues that have been identified by the current literature review. The majority of the studies included have been designed and conducted in US, where the surrounding conditions and needs for mental health care among nurses may slightly differ from those exist around the globe. These scientific data and knowledge deriving from research on preventive programs may be usefully applied to the case of nurses in countries other than the US. However, the fact that different countries operate very different health care systems may imply that there are limitations in generalizing and integrating the study findings (Edwards and Burnard, 2003). In line with this, most authors of the identified interventions recognized the limited generalizability of their results. In fact, it was found that most of the interventions were developed for a clinical environment and in most cases, they reported a small sample size or a homogeneous study population. Nevertheless, no interventions were identified recruiting large random samples, for example, in nursing homes, elderly care or health care facilities for homeless, where nurses may have increased needs for help and be at higher risk for chronic stress and stress-related diseases. In general, research results should be generalized with caution and future studies may adjust their methods to the local conditions of health care professionals. Another issue related to generalizability of the study findings extracted is that, in many articles, $\mathrm{p}$ values were reported without including the effect size, which is a standardized measure to evaluate interventions' clinical utility. Therefore, this fact rendered the comparison of the results difficult and based on this one might question their generalizability to other settings. Although previous research on stress reduction in health care providers has also identified the same methodological problem (e.g., Edwards and Burnard, 2003; Bakker et al., 2020), it still concerns. Furthermore, future research may pay more attention into different nursing specialties by developing strategies to meet the demands of non-hospital-based institutions (Bakker et al., 2020). Another major limitation of the included studies was lack of long-term follow-up data. Although the majority of the studies effectively decreased work-related stress immediately after the intervention and highlighted the benefits of such interventions for enhancing nurse's mental health, only nine programs out of the 27 identified interventions indicated a long-term change in the measured outcome. Nurses might have shown temporary improvements in stress immediately following a stress management program but might have returned later to baseline levels, especially without continued support. Hence, future interventions are needed to include longer followup intervals that can more reliably indicate the extent of their effect.

\section{Strengths and Limitations of the Systematic Review}

The present literature review provides the reader a thorough overview of the existing programs aimed at reducing stress in 
nurses and helping them develop adequate coping resources. The benefits of these interventions examined encourage the development of clinical applications and individual-level programs toward the particular group and outcome being measured. However, there is a number of limitations of the current review that should be considered. The diversity of the interventions and their treatment characteristics hindered a comparison of the different study findings, data-pooling and meta-analysis. This limitation might be overcome by the use of a tool for the quality assessment of the extracted studies. Future authors are highly encouraged to use such appraisal instruments. For the purpose of this review, only stress programs for nurses were identified and considered for further analysis. However, other groups of health care providers, such as nurse aids, novice nurses and nursing students, suffer from significant levels of distress and mental health problems (Mackenzie et al., 2006; Pulido-Martos et al., 2012; e.g., Chatterjee et al., 2014; Rathnayake and Ekanayaka, 2016). A deeper understanding of the needs of each health professional group may explain whether and why they respond to preventive programs differently. By addressing this important issue, it may help not only to implement individually tailored interventions, but also to create a basis to allocate effectively resources for interventions that alleviate stress in health care providers at all levels. Moreover, another limitation was that literature search was restricted to articles published in English or German, which might have caused the exclusion of relevant studies. In an attempt to limit the articles identified only to those that focused on interventions for nurses, authors used the term "nurses" for conducting their literature search and as a result, they might unknowingly exclude other relevant studies. This is a limitation that should be considered.

\section{CONCLUSION}

Individual-level interventions and self-care strategies are core values for addressing the growing problem of stress among nurses. However, the question of what stress management programs would be effective to enhance nurses' personal resources to decrease stress throughout the pandemic still persists. This systematic review of the literature highlights the immediate need for evidence-based preventive interventions that may be delivered through digital technology combined with relaxation and cognitive-behavioral components to reduce stress and meet the current conditions that allow fewer human contacts. The integration of VR as a tool of stress management into mental health research has the potential to offer a radical transformation

\section{REFERENCES}

Aguilera, G. (2011). HPA axis responsiveness to stress: implications for healthy aging. Exp. Gerontol. 46, 90-95. doi: 10.1016/j.exger.2010.08.023

Alkhawaldeh, J. M., Soh, K. L., Mukhtar, F., Peng, O. C., Alkhawaldeh, H. M., Al-Amer, R., et al. (2020). Stress management training program for stress reduction and coping improvement in public health nurses: a randomized controlled trial. J. Adv. Nurs. 76, 3123-3135. doi: 10.1111/ jan. 14506 of the traditional intervention programs, allowing their users to meet the current restrictions for human contact. Therefore, special attention should be paid to advancing technologybased interventions that develop innovative self-help strategies, and to applying standardized objective measurement tools to allow the quantification of sensitive physiological indices and transferability of scientific knowledge. Further research is needed to develop preventive programs with long-term follow-up for those nurses who work in specialized care at non-hospital institutions so as to understand and meet their needs for mental health care. Nurses play an integral role in each health care system and should be provided with all these appropriate selfhelp strategies to enhance their resilience and create a healing environment, where they can better exhibit their skills and commitment to patients. Having healthy staff is essential to delivering high-quality health care and preventing serious mental health disorders at the workplace.

\section{DATA AVAILABILITY STATEMENT}

The original contributions presented in the study are included in the article, further inquiries can be directed to the corresponding author.

\section{AUTHOR CONTRIBUTIONS}

MV designed the study. MV, and GR performed the literature review, data extraction, and classification according to the pre-defined criteria. MV synthesized the study findings and drafted the manuscript. GR participated into the review process. Both authors contributed to the article, read and approved the submitted version.

\section{FUNDING}

This work was funded by the German Federal Ministry of Education and Research (BMBF) as part of the joint project Digital Angel (funding program Future of Work, funding code 02L18A081).

\section{ACKNOWLEDGMENTS}

This study was conducted within the project Digital Angel (Digitaler Engel-Stärkung der Interaktionsarbeit von Pflegekräften durch den Einsatz digitaler Assistenzsysteme) funded by the German Federal Ministry of Education and Research (BMBF). 
nurses to prevent dropout: a systematic review. J. Adv. Nurs. 76, 2494-2509. doi: 10.1111 /jan. 14453

Bernburg, M., Groneberg, D., and Mache, S. (2020). Professional training in mental health self-care for nurses starting work in hospital departments. Work 67, 583-590. doi: 10.3233/WOR-203311

Bernburg, M., Groneberg, D. A., and Mache, S. (2019). Mental health promotion intervention for nurses working in German psychiatric hospital departments: a pilot study. Issues Ment. Health Nurs. 40, 706-711. doi: 10.1080/01612840.2019.1565878

Bosma, H., Marmot, M. G., Hemingway, H., Nicholson, A. C., Brunner, E., and Stansfeld, S. A. (1997). Low job control and risk of coronary heart disease in whitehall II (prospective cohort) study. BMJ 314, 558-565. doi: $10.1136 / \mathrm{bmj} .314 .7080 .558$

Calder Calisi, C. (2017). The effects of the relaxation response on nurses' level of anxiety, depression, well-being, work-related stress, and confidence to teach patients. J. Holist. Nurs. 35, 318-327. doi: 10.1177/0898010117719207

Chatterjee, S., Saha, I., Mukhopadhyay, S., Misra, R., Chakraborty, A., and Bhattacharya, A. (2014). Depression among nursing students in an Indian government college. Br. J. Nurs. 23, 316-320. doi: 10.12968/bjon.2014.23.6.316

Chesak, S. S., Cutshall, S. M., Bowe, C. L., Montanari, K. M., and Bhagra, A. (2019). Stress management interventions for nurses: critical literature review. J. Holist. Nurs. 37, 288-295. doi: 10.1177/0898010119842693

Cohen-Katz, J., Wiley, S. D., Capuano, T., Baker, D. M., and Shapiro, S. (2005). The effects of mindfulness-based stress reduction on nurse stress and burnout, Part II: a quantitative and qualitative study. Holist. Nurs. Pract. 19, 26-35. doi: 10.1097/00004650-200501000-00008

Collier, L., Staal, J., and Homel, P. (2018). Multisensory environmental therapy (Snoezelen) for job stress reduction in mental health nurses: a randomized trial. Int. J. Altern. Complement. Med. 11:2. doi: 10.15406/ijcam.2018.11.00346

Cottrell, S. (2001). Occupational stress and job satisfaction in mental health nursing: focused interventions through evidence-based assessment. J. Psychiatr. Ment. Health Nurs. 8, 157-164. doi: 10.1046/j.1365-2850.2001.00373.x

Delgado, C., Upton, D., Ranse, K., Furness, T., and Foster, K. (2017). Nurses' resilience and the emotional labour of nursing work: an integrative review of empirical literature. Int. J. Nurs. Stud. 70, 71-88. doi: 10.1016/j.ijnurstu.2017.02.008

Eatough, E. M., Chang, C. H., Miloslavic, S. A., and Johnson, R. E. (2011). Relationships of role stressors with organizational citizenship behavior: a metaanalysis. J. Appl. Psychol. 96, 619-632. doi: 10.1037/a0021887

Edwards, D., and Burnard, P. (2003). A systematic review of stress and stress management interventions for mental health nurses. J. Adv. Nurs. 42, 169-200. doi: 10.1046/j.1365-2648.2003.02600.x

Ghawadra, S. F., Abdullah, K. L., Choo, W. Y., and Phang, C. K. (2019). Mindfulness-based stress reduction for psychological distress among nurses: a systematic review. J. Clin. Nurs. 28, 3747-3758. doi: 10.1111/jocn.14987

Ghawadra, S. F., Lim Abdullah, K., Choo, W. Y., Danaee, M., and Phang, C. K. (2020). The effect of mindfulness-based training on stress, anxiety, depression and job satisfaction among ward nurses: a randomized control trial. J. Nurs. Manag. 28, 1088-1097. doi: 10.1111/jonm.13049

Gollwitzer, P. M., Mayer, D., Frick, C., and Oettingen, G. (2018). Promoting the self-regulation of stress in health care providers: an internet-based intervention. Front. Psychol. 9:838. doi: 10.3389/fpsyg.2018.00838

Golshiri, P., Pourabdian, S., Najimi, A., Zadeh, H. M., and Hasheminia, J. (2012). Job stress and its relationship with the level of secretory IgA in saliva: a comparison between nurses working in emergency wards and hospital clerks. J. Pak. Med. Assoc. 62, S26-S30.

Hatcher, B. J., Bleich, M. R., Connolly, C., Davis, K., Hewlett, P. O., and Hill, K. S. (2006). Wisdom at Work: The Importance of the Older and Experienced Nurse in the Workplace. Available online at: http://www.rwjf.org/files/publications/ other/wisdomatwork.pdf. (accessed May 29, 2021)

Herman, J. P., McKlveen, J. M., Ghosal, S., Kopp, B., Wulsin, A., Makinson, R., et al. (2016). Regulation of the hypothalamic-pituitary-adrenocortical stress response. Compr. Physiol. 6, 603-621. doi: 10.1002/cphy.c150015

Hersch, R. K., Cook, R. F., Deitz, D. K., Kaplan, S., Hughes, D., Friesen, M. A., et al. (2016). Reducing nurses' stress: A randomized controlled trial of a web-based stress management program for nurses. Appl. Nurs. Res. 32, 18-25. doi: 10.1016/j.apnr.2016.04.003
Home, J. F., and Orr, J. E. (1998). Assessing behaviors that create resilient organizations. Employ. Relat. Today 24, 29-39. doi: 10.1002/ert.3910240405

Hsieh, H. F., Huang, I. C., Liu, Y., Chen, W. L., Lee, Y. W., and Hsu, H. T. (2020). The effects of biofeedback training and smartphone-delivered biofeedback training on resilience, occupational stress, and depressive symptoms among abused psychiatric nurses. Int. J. Environ. Res. Public Health 17:8. doi: 10.3390/ijerph17082905

Hwang, W. J., and Jo, H. H. (2019). Evaluation of the effectiveness of mobile app-based stress-management program: a randomized controlled trial. Int. J. Environ. Res. Public Health 16:21. doi: 10.3390/ijerph162 14270

Jackson, D., Firtko, A., and Edenborough, M. (2007). Personal resilience as a strategy for surviving and thriving in the face of workplace adversity: a literature review. J. Adv. Nurs. 60, 1-9. doi: 10.1111/j.1365-2648.2007.04412.x

Johnston, D., Bell, C., Jones, M., Farquharson, B., Allan, J., Schofield, P., et al. (2016). Stressors, appraisal of stressors, experienced stress and cardiac response: a real-time, real-life investigation of work stress in nurses. Ann. Behav. Med. 50, 187-197. doi: 10.1007/s12160-015-9746-8

Jones, J. G. (1987). “Stress in psychiatric nursing”, in Stress in health professionals, eds R. Payne and J. Firth-Cozens (Chichester: Wiley), 189-209.

Kramer, V., Papazova, I., Thoma, A., Kunz, M., Falkai, P., Schneider-Axmann, T., et al. (2020). Subjective burden and perspectives of German healthcare workers during the COVID-19 pandemic. Eur. Arch. Psychiatry Clin. Neurosci. 271, 271-281. doi: 10.1007/s00406-020-01183-2

Kurebayashi, L. F. S., Gnatta, J. R., Borges, T. P., and de Silva, M. J. P. (2012). Applicability of auriculotherapy in reducing stress and as a coping strategy in nursing professionals.. Rev. Lat. Am. Enfermagem 20, 980-987. doi: 10.1590/S0104-11692012000500021

Lai, J., Ma, S., Wang, Y., Cai, Z., Hu, J., Wei, N., et al. (2020). Factors associated with mental health outcomes among health care workers exposed to coronavirus disease 2019. JAMA Netw. Open 3: e203976. doi: 10.1001/jamanetworkopen.2020.3976

Lary, A., Borimnejad, L., and Mardani-Hamooleh, M. (2019). The impact of a stress management program on the stress response of nurses in neonatal intensive care units: a quasi-experimental study. J. Perinat. Neonatal Nurs. 33, 189-195. doi: 10.1097/JPN.0000000000000396

Lazarus, R. S., and Folkman, S. (1984). Stress, appraisal, and coping. New York: Springer

Li, H., Zhao, M., Shi, Y., Xing, Z., Li, Y., Wang, S., et al. (2019). The effectiveness of aromatherapy and massage on stress management in nurses: a systematic review. J. Clin. Nurs. 28, 372-385. doi: 10.1111/jocn.14596

Lin, L., He, G., Yan, J., Gu, C., and Xie, J. (2019). The effects of a modified mindfulness-based stress reduction program for nurses: a randomized controlled trial. Workplace Health Saf. 67, 111-122. doi: $10.1177 / 2165079918801633$

Mackenzie, C. S., Poulin, P. A., and Seidman-Carlson, R. (2006). A brief mindfulness-based stress reduction intervention for nurses and nurse aides. Appl. Nurs. Res. 19, 105-109. doi: 10.1016/j.apnr.2005.08.002

Matousek, R. H., Dobkin, P. L., and Pruessner, J. (2010). Cortisol as a marker for improvement in mindfulness-based stress reduction. Complement. Ther. Clin. Pract. 16, 13-19. doi: 10.1016/j.ctcp.2009. 06.004

McElligott, D., Holz, M. B., Carollo, L., Somerville, S., Baggett, M., Kuzniewski, S., et al. (2003). A pilot feasibility study of the effects of touch therapy on nurses. J. N. Y. State Nurs. Assoc. 34, 16-24.

McEwen, B. S. (1998). Stress, adaptation, and disease: allostasis and allostatic load. Annal. New York Acad. Sci. 840, 33-44. doi: 10.1111/j.1749-6632.1998.tb09546.x

Moeini, B., Hazavehei, S. M. M., Hosseini, Z., Aghamolaei, T., and Moghimbeigi, A. (2011). The impact of cognitive-behavioral stress management training program on job stress in hospital nurses: applying PRECEDE model. J. Res. Health Sci. 11, 114-120.

Moher, D., Liberati, A., Tetzlaff, J., Altman, D. G., The PRISMA Group (2009). Preferred reporting items for systematic reviews and meta-analyses: the PRISMA Statement. PLoS Med. 6:e1000097. doi: 10.1371/journal.pmed1000097

Moustaka, E., and Constantinidis, T. C. (2010). Sources and effects of work-related stress in nursing. Health Sci. J. 4, 210-216. 
Nazari, F., Mirzamohamadi, M., and Yousefi, H. (2015). The effect of massage therapy on occupational stress of Intensive Care Unit nurses. Iran J. Nurs. Midwifery Res. 20, 508-515. doi: 10.4103/1735-9066.161001

Niva, S.ekar, L., Manikandan, A., K, M., T, G., Shriraam, V., et al. (2020). Mahamantra chanting as an effective intervention for stress reduction among nursing professionals-a randomized controlled study. Advances in Integrative Medicine.

Orly, S., Rivka, B., Rivka, E., and Dorit, S. E. (2012). Are cognitive-behavioral interventions effective in reducing occupational stress among nurses? Appl. Nurs. Res. 25, 152-157. doi: 10.1016/j.apnr.2011.01.004

Palumbo, M. V., Wu, G., Shaner-McRae, H., Rambur, B., and McIntosh, B. (2012). Tai Chi for older nurses: a workplace wellness pilot study. Appl. Nurs. Res. 25, 54-59. doi: 10.1016/j.apnr.2010.01.002

Prado, J. M. D., Kurebayashi, L. F. S., and Silva, M. (2018). Experimental and placebo auriculotherapy for stressed nurses: randomized controlled trial. Rev. Esc. Enferm. USP. 52:e03334. doi: 10.1590/s1980-220x2017030403334

Pulido-Martos, M., Augusto-Landa, J. M., and Lopez-Zafra, E. (2012). Sources of stress in nursing students: A systematic review of quantitative studies. Int. Nurs. Rev. 59, 15-25. doi: 10.1111/j.1466-7657.2011.00939.x

Rathnayake, S., and Ekanayaka, J. (2016). Depression, anxiety, and stress among undergraduate nursing students in a public University in Sri Lanka. Int. J. Car. Sci. 9, 1020-1032.

Ricci-Cabello, I., Meneses-Echavez, J. F., Serrano-Ripoll, M. J., Fraile-Navarro, D., de Roque, M. A. F., Moreno, G. P., et al. (2020). doi: 10.1101/2020.04.02.20048892

Riva, G., Bernardelli, L., Browning, M., Castelnuovo, G., Cavedoni, S., Chirico, A., et al. (2020). COVID Feel good-an easy self-help virtual reality protocol to overcome the psychological burden of coronavirus. Front. Psychiatry 11:563319. doi: 10.3389/fpsyt.2020.563319

Riva, G., and Riva, E. (2020). COVID feel good: a free vr self-help solution for providing stress management and social support during the COVID-19 pandemic. Cyberpsychol. Behav. Soc. Netw. 23, 652-653. doi: 10.1089/cyber.2020.29195.ceu

Schnall, P. L., Schwartz, J. E., Landsbergis, P. A., Warren, K., and Pickering, T. (1992). Relation between job strain, alcohol, and ambulatory blood pressure. Hypertension 19, 488-494. doi: 10.1161/01.HYP. 19.5.488

Shirey, M. R. (2006). Stress and coping in nurse managers: two decades of research. Nurs. Econ. 24:193.
Singh, R. K., and Jain, M. (2017). Efficacy of self-management techniques in reducing perceived occupational stress among nursing staff. Ind. J. Positiv. Psychol. 8:360-365.

Tyler, P., and Cushway, D. (1995). Stress in nurses: The effects of coping and social support. Stress Med. 11, 243-251. doi: 10.1002/smi.2460110140

Velana, M., and Rinkenauer, G. (in press). "Developing a methodology to examine psychophysiological responses during stress exposure and relaxation: an experimental paradigm," in ICHPS 2021: 23th International Conference on Health Psychology and Stress, Istanbul, Turkey, (accessed Nov 7, 2021).

Villani, D., Grassi, A., Cognetta, C., Toniolo, D., Cipresso, P., and Riva, G. (2013). Self-help stress management training through mobile phones: an experience with oncology nurses. Psychol. Serv. 10, 315-322. doi: 10.1037/a0026459

Walker, M. J. (2006). The effects of nurses' practicing of the HeartTouch technique on perceived stress, spiritual wellbeing, and hardiness. J. Holist. Nurs. 164-175. doi: $10.1177 / 0898010105283343$

Wang, S. C., Wang, L. Y., Shih, S. M., Chang, S. C., Fan, S. Y., and Hu, W. Y. (2017). The effects of mindfulness-based stress reduction on hospital nursing staff. Appl. Nurs. Res. 38, 124-128. doi: 10.1016/j.apnr.2017.09.014

Yang, J., Tang, S., and Zhou, W. (2018). Effect of mindfulness-based stress reduction therapy on work stress and mental health of psychiatric nurses. Psychiatr. Danub. 30, 189-196. doi: 10.24869/spsih.2018.189

Zautra, A. J., Hall, J. S., and Murray, K. E. (2010). "Resilience: a new definition of health for people and communities," in Handbook of Adult Resilience, eds J. W. Reich, A. J. Zautra, and J. S. Hall (New York, NY: The Guilford Press), 3-29.

Zhang, X., Bachmann, P., Schilling, T. M., Naumann, E., Schachinger, H., and Larra, M. F. (2018). Emotional stress regulation: the role of relative frontal alpha asymmetry in shaping the stress response. Biol. Psychol. 138, 231-239. doi: 10.1016/j.biopsycho.2018.08.007

Conflict of Interest: The authors declare that the research was conducted in the absence of any commercial or financial relationships that could be construed as a potential conflict of interest.

Copyright (c) 2021 Velana and Rinkenauer. This is an open-access article distributed under the terms of the Creative Commons Attribution License (CC BY). The use, distribution or reproduction in other forums is permitted, provided the original author(s) and the copyright owner(s) are credited and that the original publication in this journal is cited, in accordance with accepted academic practice. No use, distribution or reproduction is permitted which does not comply with these terms. 